\title{
Simultaneous Electricity Price and Demand Forecasting in Smart Grids
}

\author{
Vahid ASGARI $^{1, *}$, Ali Rahmani LARMAI ${ }^{2}$ \\ ${ }^{1}$ Mazandaran Electricity Distribution Company,Sari,Iran \\ Asgari.vahid@yahoo.com, 989352816737 \\ ${ }^{2}$ Mazandaran Electricity Distribution Company,Sari,Iran \\ Arahmani.larma@gmail.com, 989113548529
}

\begin{abstract}
Today's competitive world and economy is heavily dependent on electrical energy. Since electricity cannot be stored and producing more or less than the required needs may lead to some losses. The electric charge forecasting and pricing are considered as the main factors in planning and decisionmaking for future development plans and operation of power systems. In the future smart grids, electricity consumers will be able to react to changes in electricity prices. The total response of consumers to price could potentially shift the demand curve in the market. As a result, prices may vary from original projections. In this paper, a forecasting framework is proposed that offers such dynamics in predicting the electricity price demand. In this framework, a mechanism based on principles of data mining data mining to determine the patterns in response to changes in consumer demand and prices are used. In this model, the weather conditions (temperature and humidity), the days and special holidays are considered. And the results are expected to be done hourly, daily. Simulation results of the proposed method for forecasting demand and prices, which were obtained using the Australian electricity market, indicates that error is less compared to the previous methods.
\end{abstract}

Keywords: Data mining, forecasting, demand and prices, smart grid, energy market.

\section{Introduction}

In systems with vertically integrated structure, the production, transmission and distribution of electrical energy is exclusively controlled by the Government or an institution exclusively, that monitors the market and even determines exclusive monopoly prices. In not restructured power systems, consumers purchased energy from distribution companies that were exclusive. The consumers purchased energy with prices of just predetermined by the operating system. Manufacturers produced the power with the prices determined only by the operating system and contract and tariffs.. In these systems, power grids casualties were very high. With the restructuring of electricity as a commodity in competitive electricity markets, consumers have direct access to local distribution systems, and can choose the manufacturer.

Electricity markets are out of state control. And operate independently with independent market operator [2].

Fig (1) shows an overview of a restructuring of the electricity market. This model of economic prospects is the most satisfying, because the energy of the interaction of market price is determined. The implementation of this model requires an smart distribution grid implemented using significant amounts of telecommunication devices, measurement and data processing [3] 


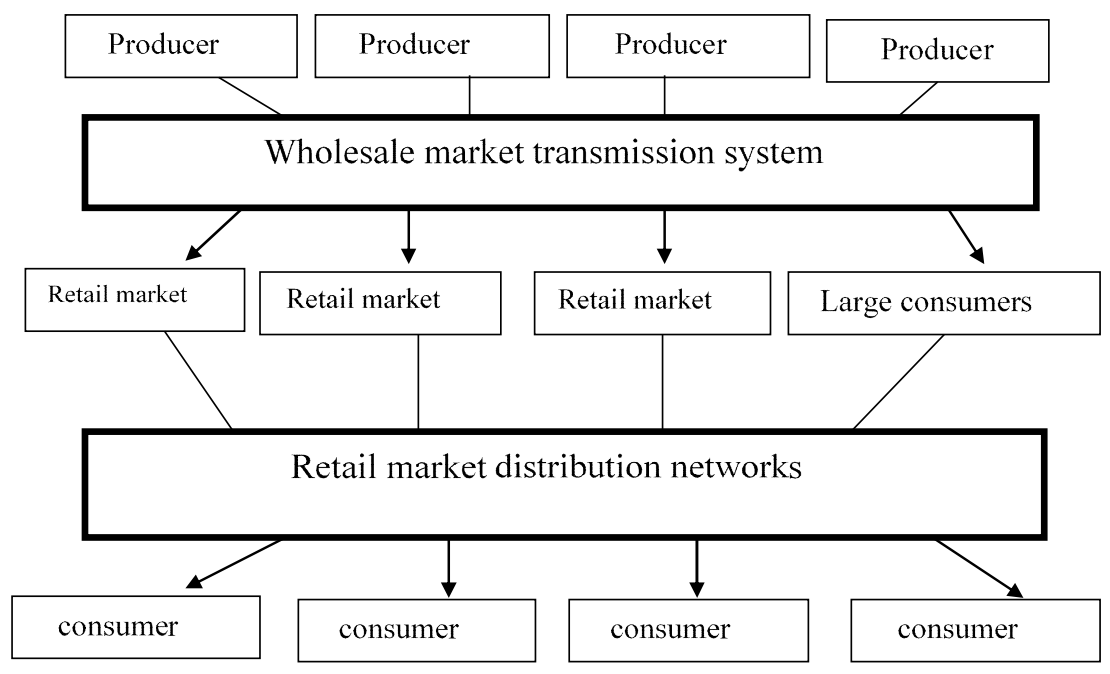

Fig 1: Model the competitive retail electricity market based on Shuttleworth and Hunt [2].

\section{Simultaneous price and demand forecasting in smart power distribution grid}

With the advent of smart electricity distribution grids, Simultaneous price and demand forecasting in the market is facing serious challenges. Predicting the price and demand for intelligent distribution grids, due to the influence of various factors such as non-linear climate changes daily and weekly and periodic dependence of the price and demand to each other is very complex.

In competitive electricity markets, the price of electricity is one of the key factors determining short-term action plans and strategies. So, data-driven approaches to model the pre-forecast electricity prices in the market have been proposed. The forecast error is about 5 to 36 percent. The extent of error due to different methods used for prediction and analysis of the market is different. The range of error in comparison with the short-term charge forecast error which is about 1 to $3 \%$, is high [4].

Various factors cause the models reduced accuracy to predict the price of electric energy. Forced outages of unpredictable generating units, complex and variable cost models, integrating energy sources, enhancing the reliability of the model are all factors that one cannot propose accurate and continuous and the same model for the volatility of price change of the electrical energy [5].

It can be seen that in the existing models, the price of electrical energy in the coming hours and the actual price formation (moment) be forecasted. However, all market participants in the decisionmaking process itself do not need to know the exact amount of future prices. For example, after the introduction of smart grid technology and new design market it is expected by interacting with the demand by households, businesses and industries a large presence in the market be appeared. Due to the nature of many charges of electric power, especially in the residential area, when the price of a certain value (threshold) is higher, demand-side market participants may initially react. But beyond that, if prices go higher, it will be considered unimportant for the consumer. Although the price is too expensive and consumers, still they consume electricity. In addition, it is possible, in many applications of response charge, a certain price for a certain time is considered. And the market price is lower than the cost of production [5].

In these types of applications that the exact amount of the price is not a priority. We can predict the price as a matter of interest to know that the price in the future.

Some research are based on the classification method can replace short-term price forecast. Classification in price, the forecast is based on whether the price according to the available objectives and programs, is above the defined threshold for the use or not [5]. 
In smart distribution girds, electrical power consumers should be able to react to energy prices. What this survey follows is that to study the influence of the price and demand, and to study the ways to improve the forecasting results in analyzing the price and demand. The main reason for having a one-sided approach to the predict price and demand in traditional gird, is the traditional elasticity of demand to the price of electricity is very low or even zero. Price sensitivity of demand, according to equation (1) is determined. Where $\mathrm{P}$ is the Price, $\mathrm{D}$ is the elasticity of demand [3].

$$
\varepsilon=\frac{D}{P} \frac{\mathrm{dP}}{\mathrm{dD}}
$$

Data-driven methods to predict the number of short-term electricity market prices and demand are presented. Now, what can be said about the expected price is a one-way relationship between price and demand. In other words, to predict the price, we assume that initially predicted a relatively "accurate at the time of the next application is available. Then the demand forecast as input models to predict prices will be used. Although many previous methods, had no demand and price forecast, they were not able to see influence of the demand in forecasting prices. Demand forecasting is in the same way. Prices influences consumer behavior and demands. In fact, in most proposed methods of demand forecasting, impacts on prices have not been anticipated in the future. The impacts of price have been considered only in rare cases [6].

However, with the development of intelligent grids, electricity consumers will be able manage their consumption in regard to economic purposes or reliability or environmental reasons. Development of advanced metering infrastructure, intelligent grids facilitates the move towards dynamic pricing and non-stationary electricity tariffs. In addition, the expected behavior of the consumer, corporate and incentive programs for consumers grow. New technologies will enable domestic consumers to use less cost time periods. Electricity demand is so ready to move from traditional inelastic patterns of behavior toward the environment of "price - response". In such an environment, two-way interactions to predict where prices and demand sides are considered will be necessary [6].

Therefore, for an accurate prediction of price and demand, in addition to factors like temperature (T), humidity (H), holiday or no holiday (d), the effects of price and demand should be considered. In this case, the price-demand relationship will be (2) and (3):

$$
\begin{gathered}
D=S^{-1}(P, T, H, d) \\
P=S(D, T, H, d)
\end{gathered}
$$

Methods that have been employed for forecasting were traditional methods based on statistical information. Advanced techniques that are based on artificial intelligence and fuzzy logic Hybrid methods for improving the results of combining several different used methods.

Generally, the amount of demand is used to predict prices price. Other inputs such as certain hours, shadow prices, transmission constraints, the exchange with neighboring systems, capacity, utilization of storage systems, hydroelectric power generating units and emergency exits are also intended to predict prices. It should be noted that the introduction of any of the aforementioned inputs does not improve results. In addition, many useful inputs to predict the price are not available [7].

For demand forecasting, demand history and background variables such as temperature, humidity, weather forecast, mainly are used as inputs of prediction models. Other variables such as time, day, week, months, and year are considered as inputs, too. A number of papers presented to forecast demand, the price in the process of forecasting are also considered [8].

Hybrid models for electricity price forecasting using time series, linear and non-linear behavior of a mixing of electricity prices in [9] and [10] have been proposed. Linear Autoregresive Integrated Moving Average or (ARIMA) and nonlinear neural grid to improve results in the Australian electricity market, is suggested in the article reference [11]. In the first step, the method proposed in the paper, ie ARIMA is used to model the behavior of prices. 
The reference paper [10], a hybrid structure to improve the prediction and update the price according to the various branches of the price is suggested. In the paper [13], high volatility and complexity of time series in the bus charges is focused. And the two-step method is proposed for prediction.

\section{The proposed method}

As stated earlier. This research is to predict the impact of smart distribution grid in real-time price and demand, by considering other factors affecting the demand and price. First, some definitions are given. Then, the proposed method is described.

\subsection{Smart grid}

One of the characteristics of smart distribution grids, by definition of IEEE is that, with the use of new technology, two-way communication between the production, supply and energy consumption will be created. The main feature of the smart grid can be active participants in the electricity market and consumers. Measuring systems with two-way communication between the customer and the market operator are used for consumer data collection and analysis in order to manage the grid to provide information for dynamic pricing.

\subsection{Data Mining}

The term "Data Mining", is the concept of unseen data mining or patterns and relationships in large volumes of data in one or more large database. Many companies and organizations have massive amounts of information. Historical data mining techniques are developed in such a way that they can be easily adapted to modern software tools, and the data collected can be used [1].

\subsection{The data mining algorithms}

Data mining algorithm, is the algorithm that detects iterative relationship between the values in a database. This algorithm has the ability to extract data without imposing any constraints on the communication link between them. Data mining is not exclusive to collect and manage data and it includes information analysis and forecasts.

\subsection{Data Mining Tools}

The most popular data mining tools in order of popularity are:

1-SPSS Modeler (data mining software developed by IBM.), 2- Rapid Miner, 3- Weka software, 4data mining software produced by Microsoft (Microsoft Analysis Services), 5-software produced by Oracle Data Mining ( Oracle Data Mining).

\subsection{Application of data mining in forecasting demand and prices}

In data mining, the general rule is that the relationship between the different values in the database is a cause and effect in the following form:

Introduction (left) $>$ the result (right).

The concept of this equation is that if the introduction or the left side happens, then the result or right side should occur. Every regulated relationship is examined by a number of quantitative measures. The two most commonly used criterion measures are "support" and "confidence", respectively [6]. Consider a database of $\mathrm{D}$ containing $\mathrm{n}$ transactions and each transaction be 
composed of a number of items, if any item by $I=\{i 1, i 2, \ldots$, in $\}$ be shown, transaction $T$ is in fact a non-empty subset of I which would be physically occurr [6].

$\mathrm{A}$ and $\mathrm{B}$ are subsets of $\mathrm{I}$ as $\mathrm{A} \Rightarrow \mathrm{B}$ are: $A \cap B=\varnothing$

"Support" is a measure for the strength of the relationship and rule discovery. As part of the transaction, that includes all of the items available, support will be determined by the following relations:

$$
\begin{aligned}
& \sup (A \Rightarrow B)=\sup (A \cup B) \\
& \sup (A \Rightarrow B)=\frac{\|\{T \in D \mid(A \cup B) \subseteq \mathrm{T}\}\|}{\|\{T \in D \mid(A) \subseteq \mathrm{T}\}\|}
\end{aligned}
$$

Where represents the number of transactions, which includes A and B. Support of a rule shows how many times the rule occurred in the studied series. In other words it indicates that the probability of introduction and conclusion, based on what the rule is.

"Reliability" is the measure of confidence in the data that is defined as follows:

$$
\begin{gathered}
\operatorname{con}(A \Rightarrow B)=\frac{\sup (A \cup B)}{\sup (A)} \\
\operatorname{con}(A \Rightarrow B)=\frac{\|\{T \in D \mid(A \cup B) \subseteq \mathrm{T}\}\|}{\|\{T \in D \mid(A) \subseteq \mathrm{T}\}\|}
\end{gathered}
$$

As shown in equation (8), Reliability of a rule, indicates the occurrence of the conclusion after its introduction [6].

For a firm and acceptable rule, acceptable minimums for support and reliability are defined in min_sup and min_con. However, these values are effectively able to demonstrate the dependence of the introduction and conclusion and the result of a rule [6].

As an alternative, the indicator "increase" or (lift) with index "support" and "confidence" is used as follows:

$$
\begin{array}{r}
\operatorname{lift}(A \Rightarrow B)=\frac{\operatorname{con}(A \Rightarrow B)}{e_{-} \operatorname{con}(A \Rightarrow B)} \\
e_{-} \operatorname{con}(A \Rightarrow B)=\frac{\sup (A) * \sup (B)}{\sup (A)}=\sup (B)
\end{array}
$$

Where and are defined as observed and expected reliability. The size of "lift" is a non-negative integer greater than 1 indicates that the results observed from the results that were expected on the basis of introduction are lower. Like expected minimum, for "reliability" and "support" a minimum "lift" is defined [6].

\subsection{The data mining process}

Data Mining includes 2 main stages. The first step finds repeated items. If "support" items for items greater than or equal to the "minimum support", these items are considered as duplicate items.

In the second phase, data mining is used for each repeated item of all rules on the relationship between the items. For each dimension of "confidence" and "lift" and those calculated above minimum values defined will be elected as the accepted rules [6]. 


\subsection{Description of the proposed method}

The proposed method to predict hourly day ahead price and demand, given the demand-side response and interaction of price and demand for intelligent electricity distribution grids are summarized as follows:

i - Price forecasting: the database of the information contained in the smart grid electricity market prices, demand, temperature, humidity, holiday or no holiday any time of the day is provided, then the information about the day ahead of time is considered including temperature and humidity levels anticipated to be derived from a reliable weather sites are extracted. Holiday or business day of the day will be determined through calendar. By Data Mining with the information contained in the database, the price is expected to be considered at the specified time.

ii - Demand forecasting: considering the above factors and importing projected prices and data mining, the demand will be forecasted.

In each of the above steps, data mining software features designed by IBM called "IBM -SPSS" were used. Applications of software are in the paper [17] and [19].

\section{The obtained results using the proposed method}

Using the data from the Australian electricity market and weather information from the internet website address included in references [13], forecasting demand and price is done. And the results are compared with the actual value.

In this study, through the proposed method and consideration of the impacts of prices and demand on each other, price and demand for a particular day, hour like 01/07/2014, was predicted. And the error rate of the proposed method was calculated. Area of "NSW1" in Australia, the electrical market has an intelligent power distribution grid. Through information from the URL mentioned in reference [13], price and hourly demand between 2013 and 2014 were identified. Meteorological data such as temperature and humidity at the time of the area in terms of Meteorology website address in the reference [14] were obtained. The Gregorian calendar, holidays and other holidays were also extracted. The information in the form of an Excel database format was arranged and classified (Table 1).

Using the software "IBMSPSS" version 22 and all the data from the above-mentioned intelligent power distribution grid and by using the proposed algorithm, prices and demand were predicted. To forecast price, variable "temperature", "humidity", "holiday or ordinary days of forecasting" and "demand" are considered as the dependent variables. to forecast demand, variables of "temperature", "humidity" "holiday or ordinary days of forecasting" and "price" are considered as the dependent variables.

Table 1: Sample Data In The Electricity Market Australia Nsw 1 On 07/01/2014

\begin{tabular}{|c|c|c|c|c|c|}
\hline Hour & Demand & Price & Temperature & Humidity & $\begin{array}{c}\text { Holiday1/ } \\
\text { non-holiday 0 }\end{array}$ \\
\hline 0 & 8625.13 & 49.39 & 10.3 & 64 & 0 \\
\hline 1 & 8239.23 & 55.69 & 10.3 & 66 & 0 \\
\hline 2 & 7795.13 & 44.9 & 10 & 73 & 0 \\
\hline 3 & 7139.07 & 44.24 & 10.1 & 74 & 0 \\
\hline 4 & 6813.64 & 41.69 & 10.4 & 74 & 0 \\
\hline 5 & 6697.04 & 42.37 & 9.4 & 76 & 0 \\
\hline
\end{tabular}


Bulletin de la Société Royale des Sciences de Liège, Vol. 86, special edition, 2017, p. 509 - 517

TABLE 2: The sample price and the actual demand and the forecast 24 hours in the area 07/01/2014

\begin{tabular}{|c|c|c|c|c|c|}
\hline Hour & Real & demand (MW) & $\begin{array}{c}\text { Anticipated } \\
\text { demand (MW) }\end{array}$ & $\begin{array}{c}\text { The real price } \\
\text { (dollars per } \mathrm{MWh} \text { ) }\end{array}$ & $\begin{array}{c}\text { Price } \\
\text { Forecast (US } \\
\$ \text { in } \mathrm{MW})\end{array}$ \\
\hline 0 & & 7842.49 & 8211.78 & 49.39 & 51.08 \\
\hline 1 & & 7468.67 & 7713.69 & 55.69 & 49.41 \\
\hline 2 & & 7044.68 & 7223 & 44.9 & 47.14 \\
\hline 3 & & 6448.52 & 6708.49 & 44.24 & 43.36 \\
\hline 4 & & 6157.55 & 6247.11 & 41.69 & 42.08 \\
\hline 5 & & 6244.31 & 6329.75 & 42.37 & 48.0 \\
\hline
\end{tabular}

NSW1 Australian electricity market

\subsection{Comparison of actual and predicted values}

Figures (2) and (3) show comparison between the actual and the predicted hourly price and demand for $07 / 01 / 2014$ :

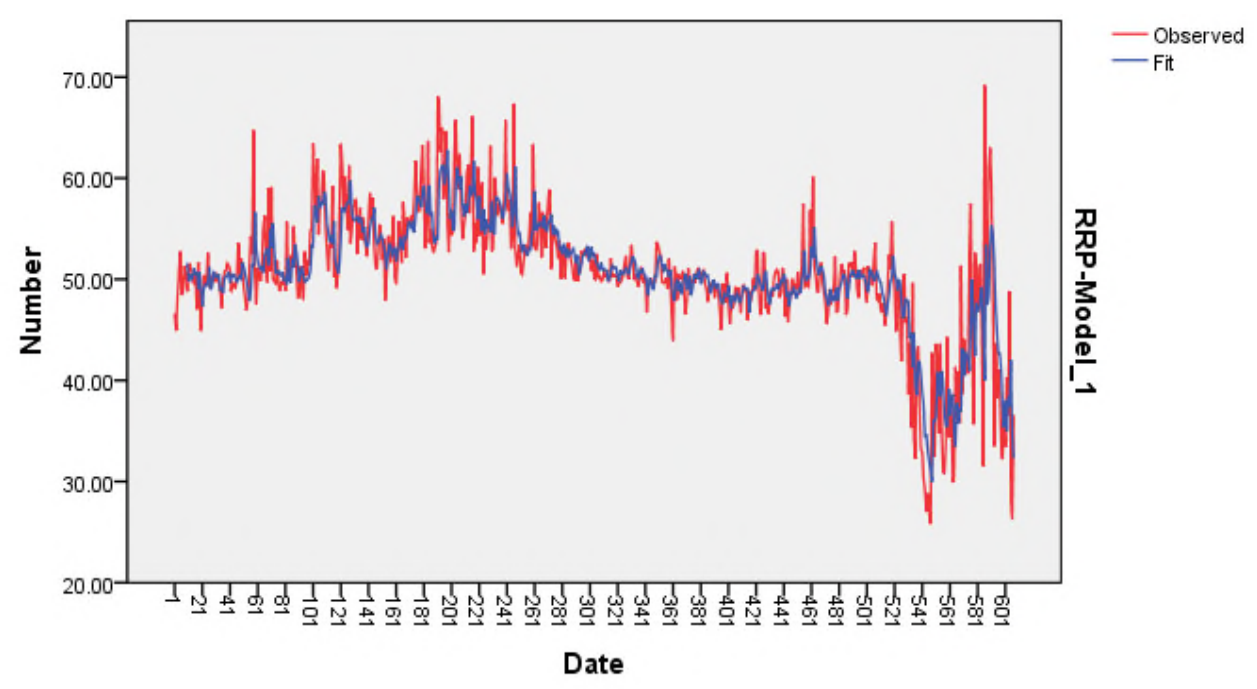

Fig 2: Charts comparing the forecast and the actual price 


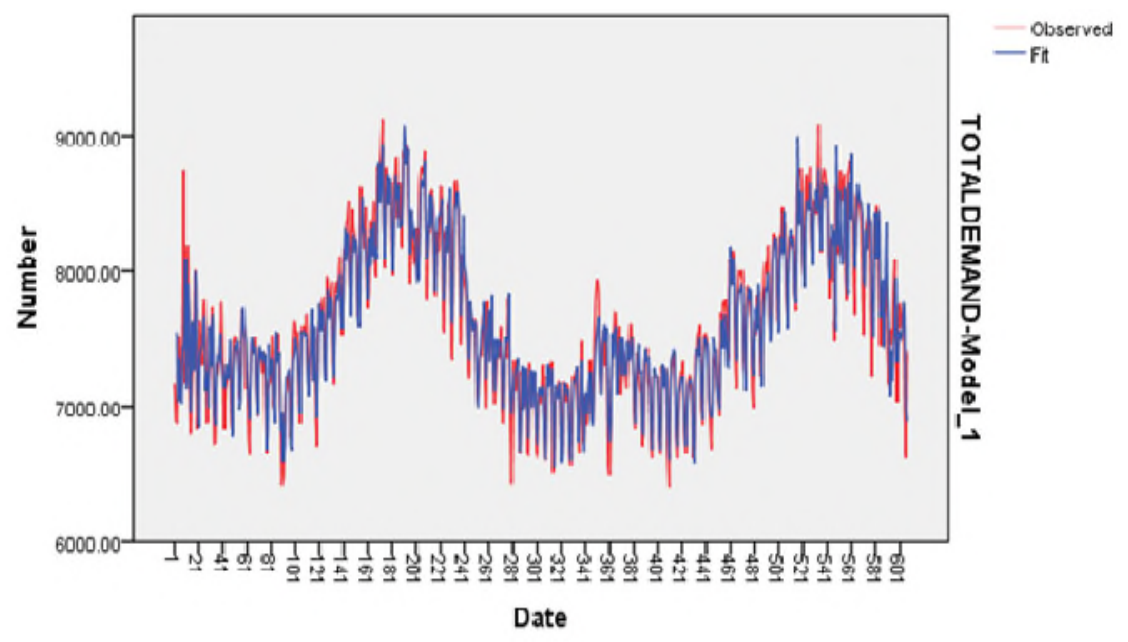

Fig 3: Comparison chart of forecast and actual demand

TABLE 3: The mean absolute percentage error (MAP) method proposed

\begin{tabular}{|l|l|l|}
\hline MEAN absolute percentage for 24 hours & Price:5.19 & Demand:1.64 \\
\hline
\end{tabular}

\section{Conclusion}

In this thesis a method to predict the price and demand for intelligent power distribution grid is presented. The price and demand are more accurately than previous methods like those in paper [6]. In [6], the mean absolute percentage error (MAPE) was 10.01 for the price and 2.31 for demand. In this article it is 5.19 and 1.64 .

The method proposed in this thesis can predict the price and demand in a smart electricity distribution network accurately. To do such, the availability of large databases with data and software and a powerful data mining is required. Therefore, it to do the same studies, a large range of data preparation and powerful software is recommended.

\section{References}

[1] www.Wikipedia.org

[2] Steven. Electric power system market economy, translated by Rahim Milani, 1389 Daniel S. Kirschen, Goran Strick, the economic fundamentals of power system. Translation by doctor Hassan monsef, Tehran, 1390

[3] Hamidreza Zareipour, Senior Member, IEEE, Arya Janjani, Student Member, IEEE, Henry Leung, Member, IEEE, Classification of Future Electricity Market Price.

[4] Cheng-Ming Lee a, Chia-Nan Ko Short-term load forecasting using lifting scheme and ARIMA models.

[5] Amir Motamedi, Member, IEEE, Hamidreza Zareipour, Senior Member, IEEE, and William D. Rosehart, Senior Member, IEEE Electricity Price and Demand Forecasting in Smart Grids.

[6] Nima Amjadya, Ali Daraeepourb, Mixed price and load forecasting of electricity markets by a new iterative prediction method

[7] L. Wu and M. Shahidehpour, "A hybrid model for day-ahead price forecasting," IEEE Trans. Power Syst., vol. 25, no. 3, pp. 519-1530, 2010

[8] J. H. Zhao, Z. Y. Dong, X. Li, and K. P. Wong, "A framework for electricity price spike analysis with advanced data mining methods," IEEE Trans. Power Syst., vol. 22, no. 1, pp. 376-385, Feb. 2007. 
[9] N. Amjady, "Short-term bus load forecasting of power systems by a new hybrid method," IEEE Trans. Power Syst., vol. 22, no. 1, pp. 333-341, Feb. 2007.

[10] P. Areekul, T. Senjyu, A. Toyama, and H. Yona, "A hybrid ARIMA and neural network model for short-term price forecasting in deregulated market," IEEE Trans. Power Syst., vol. 25, no. 1, pp. 524-530, 2010.

[11] J. Han and M. Kamber, Data Mining: Concepts and Techniques, 2nd ed. San Mateo, CA: Morgan Kaufmann, 2006.

[12] Australian Energy Market Operator [Online]. Available: http://www.aemo.com.au

[13] http://www.wunderground.com/weatherstation

[14] S.C.chan, K.M.Tsuei, H.C.Wu, Yunhe Hou, Yik-chung Wu and Felix F.Wu, LoadPrice Forecasting and Managing Demand Response for Smart Grids Methodologies and challenges, 2012.

[15] Nima Amjady, Ali Daraeepour, Mixed price and load forecasting of electricity markets by a new iterative prediction method, 2009.

[16] M.R. Braun, H. Altan, S.B.M. Beck, Using regression analysis to predict the future energy consumption of a supermarket in the UK, Applied Energy, Volume 130, 1 October 2014, Pages 305-313, ISSN 0306-2619.

[17] Rajesh V. Kale, Sanjay D. Pohekar, Electricity demand and supply scenarios for Maharashtra (India) for 2030: An application of long range energy alternatives planning, Energy Policy, Volume 72, September 2014, Pages 1-13, ISSN 0301-4215.

[18] Neil Johnson, Jian Kang, Elizabeth Abigail Hathway, Acoustics of weirs: Potential implications for micro-hydropower noise, Renewable Energy, Volume 71, November 2014, Pages 351-360, ISSN 0960-1481. 